\title{
Estudo para a construção de um instrumento de comportamentos acadêmico-sociais para estudantes universitários
}

\section{Study to the construction of an instrument of academic-social behaviors to college students}

Adriana Benevides Soares*

Universidade Salgado de Oliveira - UNIVERSO, Rio de Janeiro, RJ, Brasil

Luciana Mourão**

Universidade Salgado de Oliveira - UNIVERSO, Rio de Janeiro, RJ, Brasil

Thatiana Valory dos Santos Mello***

Universidade Estácio de Sá - UNESA, Rio de Janeiro, RJ, Brasil

\begin{abstract}
RESUMO
A presente pesquisa teve como objetivo construir e validar psicometricamente um instrumento de comportamentos acadêmico-sociais para estudantes universitários. Para a coleta de dados foram utilizados um roteiro semi-estruturado para entrevistas e um questionário na fase de validação psicométrica. O processo de elaboração gerou uma lista inicial de 240 itens com base na literatura e nos depoimentos de 100 entrevistas realizadas com professores de graduação sobre este tema. Após a validação por juízes, uma versão com 100 itens associados a uma escala do tipo Likert foi aplicada a 559 universitários de diferentes cursos no Estado do Rio de Janeiro oriundos de oito instituições de ensino superior, sendo três públicas e cinco privadas. A análise de componentes principais com rotação varimax indicou estrutura final com 34 itens e 6 fatores, a saber: comportamento adequado em sala de aula, comportamento indisciplinado em sala de aula, cordialidade no relacionamento interpessoal, desrespeito a professores e colegas, auto-exposição e assertividade, comportamento em eficácia acadêmica. Estabeleceu-se como critério de permanência itens com carga fatorial mínima de 0,30 em um único fator. Os fatores apresentaram índices de consistência interna (Alpha de Cronbach) que variavam de 0,59 a 0,80, possibilitando recomendar essa escala para realização de estudos sobre comportamentos acadêmico-sociais em estudantes universitários.
\end{abstract}

Palavras-Chave: Comportamentos sociais, Estudantes universitários, Avaliação psicológica.

\footnotetext{
ABSTRACT

This research is aimed to construct and psychometrically validate a specific instrument able to measure academic-social behaviors from college students. We used to collect data the semi-structured script for interviews and the questionnaire in the stage of psychometric validation. The drafting process generated an initial list of 240 items based both on the literature and the testimony, on this subject, from 100 interviews with college professors. After validation by judges, a version with 100 items associated
} 
with a Likert scale was administered to 559 college students from different courses in the state of Rio de Janeiro from eight higher education institutions, three public and five private. The principal components analysis with varimax rotation indicated final structure with 34 items and 6 factors, namely, behavior in the classroom, unruly behavior in class, warmth in interpersonal relationships, disrespect to teachers and peers, self-exposure and assertiveness, effective academic behavior. Like a criterion of residence for each item the minimum factor loading of 0.30 on a single factor. The factors showed high internal consistency (Cronbach's Alpha) ranging from 0.59 to 0.80 . These results indicated this scale for the studies of social behavior in students.

Keywords: Social Skills, College Students, Psychological assessment.

Pesquisas realizadas sobre vivências acadêmicas e adaptação à universidade demonstram que mais da metade dos alunos que ingressam no curso superior revelam diversos tipos de dificuldades pessoais e acadêmicas de diferente natureza (ALMEIDA; SOARES; FERREIRA, 1999; PIRES; ALMEIDA; FERREIRA, 2000; FERREIRA; ALMEIDA; SOARES, 2001; POLYDORO; PRIMI; SERPA; ZARONI; POMBAL, 2001; CUNHA, 2004; CUNHA; CARRILHO, 2005; SOARES; GUISANDE; ALMEIDA; PÁRAMO, 2008; SOARES; NAIFF; FONSECA; CARDOSO; BALDEZ, 2009).

Muitos trabalhos sobre adaptação à universidade e vivências acadêmicas têm investigado diferentes aspectos que levariam o aluno a encontrar obstáculos a serem superados na transição do ensino médio para o ensino superior (PASCARELA; TERENZINI, 1991; 2005; PIRES; ALMEIDA; FERREIRA, 2000). Alguns se fixaram em identificar as características pessoais dos estudantes que seriam preditores para uma melhor adaptação universitária (ALMEIDA; SOARES, 2004; CARELLI; SANTOS, 1998; FERREIRA; ALMEIDA; SOARES, 2001; GRANADO; SANTOS; ALMEIDA; SOARES; GUISANDE, 2005; SANTOS; SUEHIRO; OLIVEIRA; CUNHA; CARRILHO, 2006; SOARES; GUISANDE; ALMEIDA; PÁRAMO, 2008; SOARES; ALMEIDA; DINIZ; GUISANDE, 2006; VENDRAMINI; SANTOS; POLYDORO; SBARDELINI; SERPA; NATÁRIO 2004), outros sobre o impacto que as instituições de ensino podem acarretar na vida dos estudantes (SANTOS, 2000; SOARES; POUBEL; MELLO, 2009) outros ainda nas expectativas que os estudantes elaboram para com o curso e a instituição que vão frequentar (IGUE; BARIANI; MILANESI, 2008; POLYDORO, 2000; PACHANE, 2003) além de relacionar aspectos da adaptação a um melhor rendimento acadêmico (ALMEIDA; SOARES; FERREIRA, 1999; CUNHA; CARRILHO, 2005; SANTOS, 2000).

O sucesso acadêmico do ingressante em nível superior está baseado em algumas características pessoais, tais como grau de adaptabilidade, de conhecimentos, de habilidades e de atitudes. Assim, as mudanças requeridas pelo novo contexto normalmente imprime ao ingressante transformações sociais que alteram a sua 
vida futura (GERK; CUNHA, 2006). Além disso, os autores apontam resultados de pesquisas nos quais os ingressantes apresentam maiores dificuldades interpessoais do que os estudantes universitários que estão há mais tempo neste ambiente, o que reforça a preocupação com a adaptação inicial do estudante universitário.

Considerando este contexto e a relevância do tema, o presente trabalho tem por objetivo desenvolver e validar uma escala para avaliar os comportamentos acadêmico-sociais numa população de universitários no Brasil.

\section{Referencial teórico}

As ascensões requeridas na vida acadêmica e social do ingressante concomitantemente às suas relações interpessoais têm sido interessantes objetos de estudo por parte da comunidade acadêmica (BOLSONI-SILVA, 2009). A autora analisa que diante de tanta dificuldade de adaptação do estudante universitário, seria esperado que as universidades se preocupassem em ajudá-lo neste sentido, e ressalta que são poucos os estudos conduzidos nesta direção. Almeida (1998) propõe que uma melhor compreensão do ajustamento e o sucesso acadêmico dos estudantes do nível superior podem ser referenciados através das vivências ocorridas durante o processo de transição do nível médio para o superior. As propriedades dessa transição e a adequação de um estudante ao novo escopo vão depender de seu desenvolvimento psicossocial e de recursos de apoio proporcionados pela instituição. O conhecimento das características da população de alunos é de extrema relevância tanto para a administração, como para a elaboração de regras e também para a condução dos cursos e planejamento das intervenções a serem adotadas (PARKER; SUMMERFELDT; HOGAN; MAJESKI, 2004; AZEVEDO; FARIA, 2001, 2006).

$O$ ambiente acadêmico tem sido considerado um determinante para o aprimoramento do estudante porque proporciona-Ihe a possibilidade de compartilhar, com várias pessoas, idéias e experiências inovadoras (PASCARELA; TERENZINI, 2005; GRANADO et al., 2005; ALMEIDA; SOARES, 2004). Ademais, os alunos exercem um papel fundamental posto que essa transição incide em observância à intensidade de interação entre o aluno e os recursos a ele disponibilizados, e seu prévio patamar intelectual (SOARES et al., 2008; SOARES et al., 2006).

Segundo Parker, Summerfeldt, Hogan e Majeski (2004) aspectos pessoais tais como fazer novas amizades, modificar relacionamentos já existentes com a família, ter que aprender hábitos de estudo, aprender a viver e muitas vezes conviver como adultos independentes, administrando tempo e dinheiro, são funções que por 
falta de domínio social se tornam dificuldades a serem superadas pelo estudante universitário.

A entrada no mundo acadêmico para muitos é marcada pela separação dos amigos, familiares, vizinhos e parentes (ZHANG, 2000; ZHENG; KEVIN; SAUNDERS; SHELLEY, 2002; CUNHA, 2004; SANTOS et al., 2006). A autonomia do aluno é afetada pelo estabelecimentorompimento das relações interpessoais e pela percepção que ele tem sobre o apoio emocional da família e dos amigos. Segundo Santos (2000) os alunos que têm intensas ligações parentais, são encorajados pelos pais, ainda que de longe, no seu processo de aquisição de autonomia, mantendo laços positivos, constroem uma significativa percepção de apoio e segurança em relação aos mesmos e propiciam melhor capacidade de adaptação.

Cunha (2004) associa o desenvolvimento da autonomia à autoeficácia na aprendizagem, ou seja, à medida que o estudante adquire segurança nas suas aquisições de aprendizagem e conhecimento, ele passa a se sentir encorajado para buscar novos conhecimentos e desafios facilitando novos processos cognitivos. O desenvolvimento da autonomia se relaciona diretamente com a capacidade dos universitários em criar suas estratégias e métodos de aprendizagem, com a percepção que possuem do seu próprio nível de influência nas aprendizagens efetivas.

A sensação de bem estar psicológico é gerada pelas percepções de satisfação com a vida, equilíbrio emocional, estabilidade afetiva, felicidade e otimismo. Referem-se ao quanto acreditamos em nós mesmos e nas representações que temos de nossas características pessoais, tanto no âmbito negativo, como no positivo (ALMEIDA, 1998). Santos (2000) afirma que o auto-conhecimento impacta nos níveis de motivação para o sucesso no rendimento acadêmico dos universitários, pois está associado à noção de bem estar físico e psicológico.

Para muitos autores é fundamental entender de forma mais substancial a relação interacional entre a instituição e os estudantes e as consequências resultantes desta interação (PIRES et al., 2000; ALMEIDA, 1998; ALMEIDA et al., 1999; ALMEIDA; SOARES, 2004; VENDRAMINI et al., 2004). O ingresso na universidade expõe 0 estudante a estressores específicos, como inseguranças, ansiedade e medo, todos provenientes de possíveis dúvidas e desilusões com a carreira escolhida, assim como a aquisição de novas e maiores responsabilidades (SANTOS et al., 2006).

A forma como o estudante adquire seus métodos de estudos, o conhecimento que se tem do curso, a relação que mantém como corpo docente, os diferentes estilos cognitivos e as estratégias de aprendizagem influem, em muito, na forma como vão vivenciar a universidade. $O$ aluno se sente realizado quando percebe a evolução e o progresso obtido com sua aprendizagem, com a aquisição de 
novas competências, desenvolvimento da capacidade para integração de idéias e resolução de problemas, assim como de métodos e estratégias de estudos (SADALLA, 1998).

Algumas habilidades pessoais, intelectuais e acadêmicas, tais como a percepção da identidade, as decisões sobre a carreira, a conquista e a manutenção das relações interpessoais, são imprescindíveis para o sucesso acadêmico do ingressante (SANTOS, 2000; VENDRAMINI et al., 2004; GRANADO et al., 2005).

A conjuntura universitária per si já oferece um escopo propício ao desenvolvimento de seus alunos, facilitando seus processos adaptativos concernentes à integração e às relações interpessoais, sociais e afetivas (FERREIRA et al., 2001; VENDRAMINI et al., 2004; GRANADO et al., 2005). Os alunos com melhores vivências acadêmicas apresentam um melhor desempenho acadêmico do que alunos que vivenciam experiências negativas. Quando o ingressantecalouro sente-se desajustado não consegue desenvolver bem suas interações na dinâmica dos relacionamentos interpessoais (VILLAR, 2003). Segundo Santos (2000), as implicações das vivências negativas provocam uma queda no rendimento acadêmico e aceleram um aumento no índice de abandono escolar e solicitação aos serviços psicossociais.

Sabe-se que, de forma geral, a educação superior provoca mudanças no corpo discente, tanto em nível pessoal, profissional, quanto social. Durante o período de educação superior, devido aos diversos tipos de atividades acadêmicas e educacionais, os alunos estão freqüentemente estimulados a alterarem continuamente suas experiências e vivências (PASCARELLA; TERENZINI, 2005).

Segundo Ferreira et al. (2001) e Cunha e Carrilho (2005), a noção de sucesso acadêmico está intimamente ligada às vivências dos universitários que estão no primeiro ano do curso, se distanciando da abordagem nucleada unicamente no rendimento acadêmico. Assim, o sucesso acadêmico do estudante deve ser avaliado em função do seu próprio desenvolvimento e de seus objetivos, considerando o contexto de um desenvolvimento holístico ao qual espera-se deste universitário um conjunto dinâmico de competências adaptativas a um ambiente não só novo como também dinâmico pelas suas próprias características.

Diversos pesquisadores em habilidades sociais (BANDEIRA; GUAGLIA; FREITAS; SOUZA; COSTA; GOMIDES; LIMA, 2006; BANDEIRA; GUAGLIA; FREITAS; SOUZA; COSTA; GOMIDES; LIMA, 2000; BANDEIRA; QUAGLIA, 2005; DEL PRETTE; DEL PRETTE, 1983; 2003; DEL PRETTE; DEL PRETTE; BARRETO; BANDEIRA; RIOS-SALDANÃ; ULIAN; GERK-CARNEIRO; FALCONE; VILLA, 2004; DEL PRETTE; DEL PRETTE; BARRETO, 1999; 1998; FURTADO; FALCONE; CLARK, 2003) têm se preocupado com os estudantes universitários. Em algumas de suas pesquisas buscam caracterizar as habilidades sociais dos 
universitários, o que facilita o desenvolvimento de mais pesquisas que visem à promoção dessas habilidades sociais em uma tentativa de identificar os possíveis déficits e as habilidades mais desenvolvidas para contribuir tanto na adaptação acadêmica como na inserção no mercado de trabalho, entretanto, nenhuma medida específica de habilidades sociais no contexto universitário brasileiro foi encontrada. Apesar do interesse em habilidades sociais e do crescente volume de pesquisas mostrando sua relevância, ainda há no Brasil uma carência de escalas. Bandeira, Del Prette, Del Prette e Magalhães (2009) desenvolveram as Escalas de Habilidades Sociais, Comportamentos Problemáticos e Competência Acadêmica (SSRS-BR) a qual destina-se aos alunos do Ensino Fundamental. A referida escala avalia habilidades sociais, comportamentos problemáticos e competência acadêmica de estudantes do Ensino Fundamental. O estudo de Bandeira et al. (2009) foi feito com 416 estudantes, de ambos os sexos, que estavam no primeiro ciclo do ensino fundamental ( $1^{a}$ a $4^{a}$ séries). Os resultados obtidos são bastante satisfatórios, pois a estrutura de componentes conseguiu explicar de $40 \%$ a $62 \%$ da variância dos dados. A análise da consistência interna indicou adequado grau de precisão (alfa de Cronbach) para as escalas de habilidades sociais, sendo os alphas respectivamente de 0,78, 0,86 e 0,94 , considerando o público de estudantes, pais e professores. Vale destacar que os autores também pesquisaram, além das habilidades sociais, os comportamentos problemáticos (com alphas de 0,83 (pais) e 0,91 (professores) e competência acadêmica $(0,98)$. Comprovando a estabilidade temporal do instrumento, as correlações teste-reteste foram positivas e significativas para os escores globais das escalas de habilidades sociais (correlação igual ou superior a 0,70 em todos os casos).

Este instrumento, embora muito interessante e englobando competência acadêmica e habilidades sociais no ambiente acadêmico, está voltado para o ensino fundamental, sendo necessário instrumentos que se destinem ao público adulto. Assim, levando-se em consideração a necessidade de instrumentos para avaliar as comportamentos acadêmico-sociais no âmbito universitário, principalmente aqui no Brasil, realizou-se a presente pesquisa cujo método é relatado a seguir.

\section{Método}

Nesta seção são apresentados e discutidos os processos de elaboração e validação - semântica, teórica e estatística - do instrumento que busca auferir comportamentos acadêmico-sociais de estudantes no contexto universitário. São detalhados tanto os procedimentos para a criação da escala, como aqueles que atestam a validade do instrumento. 
Os itens do instrumento de razões para participar de programas sociais foram desenvolvidos a partir das suposições inerentes à revisão de literatura de habilidades sociais, levando-se em conta, sobretudo os estudos de Del Prette et al. $(1998$; 1999) e de Almeida e Ferreira (1997), respectivamente em relação ao Inventário de Habilidades Sociais - IHS e ao Questionário de Vivências Acadêmicas - QVA.

Participantes

Participaram da pesquisa 559 estudantes universitários no Estado do Rio de Janeiro, oriundos de oito instituições de ensino superior, sendo três públicas e cinco privadas. Os participantes estavam matriculados em diferentes cursos, a saber: Administração, Biologia, Direito, Enfermagem, Engenharia Mecânica, Farmácia, Matemática, Pedagogia, Processamento de Dados, Psicologia, Química e Serviços Sociais, com distribuição dos respondentes pelos diversos semestres dos cursos de graduação.

A participação de cada um desses sujeitos foi voluntária e eles assinaram o Termo de Consentimento Livre e Esclarecido, tendo sido o presente projeto submetido ao Comitê de Ética da Universidade Salgado de Oliveira - UNIVERSO e por ele aprovado.

Instrumentos de Coleta de Dados

Foram utilizados dois instrumentos de coleta de dados: roteiro semiestruturado para as entrevistas e questionário para validação da Escala de Habilidades Sociais em Estudantes Universitários - HSUE. O roteiro para as entrevistas tinha apenas perguntas-tema. Em relação ao questionário, os itens estavam associados a uma escala do tipo Likert, de frequência, contendo cinco pontos, na qual o valor 1 representava nunca, e o valor 5 , sempre.

Foi realizada validação semântica com estudantes universitários, por ser essa a população escolhida para a validação do instrumento. Nessa etapa, os respondentes liam o instrumento e apontavam sugestões de melhoria na redação do mesmo em termos da clareza da linguagem empregada no desenvolvimento da versão inicial da escala. Foram feitos ajustes no instrumento e nas orientações para resposta, na redação de alguns itens e no formato do questionário.

Após a validação semântica, os itens foram submetidos à apreciação de cinco juízes, entre mestres e doutores especialistas no tema. Os fatores propostos foram: (a) Fator 1 - Reações internalizantes comportamentos apresentados pela pessoa para lidar com as demandas interativas do ambiente, por serem expressos predominantemente de forma encoberta; (b) Fator 2 - Reações externalizantes - comportamentos apresentados pela pessoa para lidar com as demandas interativas do seu ambiente, por serem expressos predominantemente de forma aberta; (c) Fator 3 - 
Reações Comportamentais - reações expressas por ações diretamente observáveis diante das demandas interpessoais; e (d) Fator 4 - Reações Não-comportamentais - reações afetivas ou cognitivas diante das demandas interpessoais.

Foram considerados válidos os itens corretamente classificados, por $80 \%$ ou mais de juízes, como pertencentes ao conceito referente. Validado semanticamente e por juízes, o instrumento ficou, então, composto por 79 itens associados à escala mencionada anteriormente e foi submetido a um processo de validação empírica. Para tanto, o instrumento foi aplicado em 559 estudantes universitários. Ao final da coleta de dados e após a retirada de casos e valores extremos, adotando-se como critério a Distância Mahalanobis, obteve-se uma amostra de 447 questionários válidos. A respeito do tamanho da amostra, a mesma mostrou-se adequada, considerando o critério mínimo de cinco questionários válidos por item da escala.

\section{Procedimentos de Coleta de Dados}

Os procedimentos de coleta de dados envolveram duas técnicas: entrevistas em profundidade e aplicação de questionários. Para a elaboração dos itens do instrumento criou-se uma lista inicial de 240 itens com base na literatura sobre comportamentos e habilidades sociais na academia e também com base nos depoimentos das 100 entrevistas realizadas com professores de graduação sobre este tema. A entrevista foi presencial, em dia e hora marcados previamente com os participantes, no período de março a junho de 2009.

A proposta durante as entrevistas era de ouvir o que as pessoas tinham a dizer sobre o que consideram comportamentos habilidosos e não-habilidosos na academia, sem direcionamento de respostas ou expressão de qualquer opinião a respeito. A adoção desse procedimento permitiu que surgissem dos entrevistados informações relevantes que deram origem aos itens do instrumento validado no presente estudo.

Procedimentos de Análise de Dados

Para o levantamento dos itens foi realizada análise de conteúdo categorial (BARDIN, 1977) nos dados qualitativos originados das entrevistas. A análise de conteúdo caracteriza-se como procedimento de fragmentação de textos com o objetivo de identificar regularidades e possibilita tratar todo o material textual, buscando identificar a pluralidade temática presente num conjunto de textos, ao mesmo tempo em que pondera a freqüência desses temas dentro do mesmo conjunto (NASCIMENTO; MENANDRO, 2006).

Neste estudo, a análise de conteúdo foi feita a partir do agrupamento de elementos de significados mais próximos, com formação de categorias e subcategorias (análise categorial). As categorias 
derivaram não apenas da relevância, mas também da recorrência dos temas no corpus dos depoimentos dos pesquisados. Para a validação psicométrica utilizou-se estatística para análise de componentes principais e análise fatorial, que permitiram a validação da Escala de comportamentos acadêmico-sociais em Estudantes Universitários CASUE. Para tanto, contou-se com o apoio do software Statistical Package for the Social Sciences - SPSS versão 15.0. para a análise de dados.

\section{Resultados}

As respostas válidas foram, então, primeiramente submetidas a análises descritivas. Os pressupostos de multicolinearidade e singularidade foram devidamente investigados. O pressuposto da normalidade, embora não seja uma exigência - por ser a análise fatorial uma técnica de análise robusta - também foi testado, pois os dados tendem a apresentar melhor configuração quando tal pressuposto é atendido.

Em seguida, foram realizadas análises preliminares para verificar a fatorabilidade da matriz de correlações e análises fatoriais exploratórias (Principal Axis Factoring) para estimar o número de fatores e definir a estrutura fatorial do instrumento. Foram considerados, para efeitos de validação, somente itens com cargas fatoriais superiores a 0,30 , teoricamente interpretáveis e com índices de confiabilidade superiores a 0,60 (em ambos os casos considerando-se os valores com arredondamento de uma casa decimal). As análises, como a quantidade de respostas em branco aos 79 itens analisados não ultrapassou a margem de $2 \%$, adotou-se o tratamento pairwise para dados omissos.

A respeito da fatorabilidade da matriz de dados, $\mathrm{o}(\mathrm{KMO}=0,83)$ e o teste de esfericidade de Bartlett $(p<0001)$ indicaram a adequação dos dados para a análise fatorial. Com a finalidade de escolher a estrutura empírica mais adequada aos dados, foram realizadas a análise do screeplot, a análise paralela e a análise da consistência teórica. O screeplot sugere a extração de dez fatores. Porém, a análise paralela, proposta por Enzmann (1997), a qual apresenta os valores aleatórios de eigenvalues para uma matriz de dados com 79 itens e 447 sujeitos, sugeriu a extração de oito fatores. De acordo com esse teste, devem ser extraídos tantos fatores quantos os eigenvalues empíricos forem superiores aos eigenvalues aleatórios.

Ao contrário do esperado, os dados não se agruparam nos fatores inicialmente planejados: Reações internalizantes $X$ Reações externalizantes e Reações Comportamentais $X$ Reações Nãocomportamentais. O agrupamento estatístico dos itens levou a uma solução de comportamentos acadêmico-sociais relativamente ao contexto e ao interlocutor ao qual os comportamentos acadêmicosociais são dirigidas em termos do ambiente universitário. Porém, 
dois fatores tiveram índices de confiabilidade muito baixos (abaixo de 0,4 ) e um agrupamento de fatores que não apresentava consistência teórica. Assim, considerados os dados psicométricos e a consistência teórica, permaneceu na escala um total de 34 itens, divididos em seis fatores, conforme apresentado na Tabela 1.

Tabela 1: Fatores de comportamentos acadêmico-sociais em universitários, com cargas fatoriais dos itens e o grau de fidedignidade de cada fator

\begin{tabular}{l|c|c|c}
\hline \multicolumn{1}{c|}{ Fatores } & Itens & $\begin{array}{c}\text { Alpha de } \\
\text { Cronbach }\end{array}$ & $\begin{array}{c}\text { Cargas } \\
\text { fatoriais }\end{array}$ \\
\hline $\begin{array}{l}\text { F1 - Comportamento adequado em } \\
\text { sala de aula }\end{array}$ & 6 itens & 0,73 & $\begin{array}{c}\text { Entre } 0,34 \mathrm{a} \\
0,63\end{array}$ \\
\hline $\begin{array}{l}\text { F2 - Comportamento indisciplinado } \\
\text { em sala de aula }\end{array}$ & 6 itens & 0,81 & $\begin{array}{c}\text { Entre } 0,43 \mathrm{a} \\
0,57\end{array}$ \\
\hline $\begin{array}{l}\text { F3 - Cordialidade no } \\
\text { relacionamento interpessoal }\end{array}$ & 6 itens & 0,77 & $\begin{array}{c}\text { Entre } 0,40 \mathrm{a} \\
0,57\end{array}$ \\
\hline $\begin{array}{l}\text { F4 - Desrespeito a professores e } \\
\text { colegas }\end{array}$ & 5 itens & 0,59 & $\begin{array}{c}\text { Entre } 0,30 \mathrm{a} \\
0,44\end{array}$ \\
\hline $\begin{array}{l}\text { F5 - Auto-exposição e } \\
\text { assertividade }\end{array}$ & 6 itens & 0,66 & $\begin{array}{c}\text { Entre } 0,36 \mathrm{a} \\
0,52\end{array}$ \\
\hline $\begin{array}{l}\text { F6 - Comportamento de eficácia } \\
\text { academica }\end{array}$ & 5 itens & 0,60 & $\begin{array}{c}\text { Entre } 0,31 \mathrm{a} \\
0,42\end{array}$ \\
\hline
\end{tabular}

No que se refere à correlação entre os dois fatores da escala, houve correlação significativa $(p \leq 0,05)$, mas com um coeficiente de Pearson apontando uma maioria de correlações bivariadas entre os fatores significativas, com coeficientes entre 0,10 e 0,46 (Tabela 2). Em função dessa correlação, utilizou-se a técnica de rotação varimax.

Tabela 2: Correlação entre os fatores da escala de habilidades sociais

\begin{tabular}{l|r|r|r|r|r}
\hline Correlação de Pearson & Fator 2 & Fator 3 & Fator 4 & Fator 5 & Fator 6* \\
\hline Fator 1 &,- 063 &, $260(* *)$ &, $108\left(\left(^{*}\right)\right.$ &, $436(* *)$ &, $360(* *)$ \\
Fator 2 & & $-288(* *)$ &, $433(* *)$ &,- 024 &, $192(* *)$ \\
Fator 3 & & & - &, $302(* *)$ &, $373(* *)$ \\
& & &, $143(* *)$ &, $169(* *)$ &,$- 099(*)$ \\
Fator 4 & & & & &, $464(* *)$ \\
Fator 5 & & & & &
\end{tabular}

* Significância ao nivel $\mathrm{p}<0,05$

** Significância ao nível $p<0,01$

A variância total explicada dos seis fatores foi de $42 \%$. Em relação à variância explicada por cada um dos fatores, como eles apresentam uma correlação significativa e moderada entre si, é provável que essa variância explicada tenha reciprocamente a influência do outro fator. Portanto, seria mais recomendável considerar apenas a variância explicada total da escala (42\%). 
Observou-se que para a formação da versão final dessa escala, o processo empírico eliminou alguns itens complexos, que tinham cargas significativas nos dois fatores e outros que não apresentavam cargas acima de 0,30 em nenhum fator. Para se ter certeza da estrutura final da escala, foram calculados os índices de precisão da escala (Alpha de Cronbach) se deletado cada um dos itens dos seis fatores extraídos. O resultado apontou que qualquer que fosse $o$ item retirado, haveria uma diminuição do grau de precisão, confirmando a pertinência de manutenção dos 36 itens da versão final da escala.

\section{Discussão e Considerações Finais}

O trabalho realizado por Bolsoni-Silva (2009) com o objetivo de comparar as habilidades sociais (IHS-Del Prette) de 39 estudantes de psicologia antes e após frequentarem um estágio supervisionado em treinamento de habilidades sociais apontou melhoras em quatro dos cinco fatores avaliados, permitindo uma rica discussão acerca do repertório social e da saúde mental dos universitários.

De fato, problemas educacionais são hoje em grande parte relativos a questões de natureza interpessoal. São encontrados na literatura relatos de problemas de relacionamento social em diversos contextos acadêmicos que incluem desde crianças pré-escolares até jovens adolescentes e adultos universitários (DEL PRETTE, 2005; VILLASBOAS SILVEIRA; BOLSONI-SILVA, 2005; FURTADO; FALCONE; CLARK, 2003; CASTILLA, 2007). Neste sentido torna-se importante a construção de instrumentos de medida que permitam a identificação de problemas de relacionamento interpessoal no contexto educacional que estejam padronizados para nossa cultura.

Embora alguns instrumentos de medida tenham sido construídos com a finalidade de servir de suporte para a identificação e diagnóstico dos repertórios de habilidades sociais (DEL PRETTE; DEL PRETTE; 2001; DEL PRETTE; PRETTE, 2002; FALCONE; FERREIRA; LUZ; FERNANDES; FARIA; D'AUGUSTIN; SARDINHA; PINHO, 2008; DEL PRETTE; VILLA, FREITAS; DEL PRETTE, 2008; DEL PRETTE; DEL PRETTE, 2009) não foi encontrado nenhum instrumento especificamente relacionado ao contexto de relacionamento interpessoal na Universidade.

Para a construção deste instrumento as análises estatísticas conduzidas, ao nível da consistência interna dos itens de cada subescala formada, bem como da sua estrutura fatorial (validade interna), permitiram valores adequados à sua utilização para uma descrição dos níveis de comportamentos acadêmico-sociais dos estudantes na Universidade. As correlações calculadas mostram, também, uma relação diferenciada dos diversos fatores avaliados.

A análise fatorial sustentou a validade de construto do teste na qual foi observado seis fatores ligados à habilidade social acadêmica em universitários. Os índices de consistência interna calculados para cada 
item em suas respectivas subescalas indicaram que os itens que compõem cada fator estão adequados para mensurar o que cada fator propõe, pois atingem o valor mínimo aceito de 0,30 mensurando de forma homogênea cada fator.

Os seis fatores encontrados (habilidades em sala de aula, comportamento indisciplinado, cordialidade, desrespeito a professores e colegas, auto-exposição e assertividade e eficácia acadêmica) representam alguns das dimensões que descrevem os comportamentos acadêmico-sociais, servindo desta forma aos objetivos pretendidos.

Os resultados da análise fatorial encontrados no presente estudo corroboram, em parte, os resultados de outros trabalhos, como por exemplo, de Bandeira et al. (2000), que identificaram cinco fatores, sendo que dois deles também puderam ser, com ressalvas, observados nesta pesquisa. Estes fatores compreendem neste instrumento a cordialidade nos relacionamentos interpessoais e autoexposição e assertividade que foram identificados em alguns itens da escala. Entretanto, no presente estudo, abordou-se comportamentos acadêmico-sociais do aluno nestes aspectos indicando comportamentos relacionais no contexto universitário. Também foi encontrado o fator Habilidade em Eficácia Acadêmica que corresponde em parte aos resultados encontrados em Sá (2006) em seu instrumento de auto-eficácia acadêmica percebida.

A análise preliminar do instrumento indica bons resultados psicométricos referentes a sua validade de construto, através de análise fatorial exploratória apresentando índices de precisão satisfatórios. As seis subescalas do instrumento representam características multidimensionais das relações interpessoais habilidosas no ambiente universitário.

As subescalas Comportamento adequado em sala de aula e Comportamento indisciplinado correspondem ao componente comportamental positivo e negativo das habilidades exigidas e esperadas em sala de aula, tal como mencionado por (PERRENOUD, 1999; 2000; 2001; PERRENOUD; GATHER THURLER; MACEDO; MACHADO; ALLESSANDRINI, 2002), os itens da subescala de Cordialidade medem reações internalizantes e externalizantes, habilidosas e não habilidosas da desenvoltura social conforme estudos revisados por (BANDEIRA; QUAGLIA, 2005; DEL PRETTE, 2003).

A subescala Desrespeito a professores e colegas, por sua vez, representa o componentes interpessoais diretos entre pares e autoridades; a subescala auto exposição e assertividade está relacionada a componentes que envolvem um certo risco de reação indesejada (CABALLO, 1982; SOARES; POUBEL; MELLO, 2009; DEL PRETTE; DEL PRETTE, 1983) e a subescala de eficiência acadêmica 
envolve itens auto regulatórios de competências e adaptação acadêmica (SÁ, 2006; SOARES et al., 2009) .

Assim, todos os fatores do instrumento representam especificidades do comportamento social universitário, o que não ocorre com os instrumentos apresentados anteriormente. Enquanto a medida de (DEL PRETTE; DEL PRETTE, 2002) se propõe avaliar competências sociais de estudantes do ensino fundamental, a de (DEL PRETTE; DEL PRETTE, 2001) mede habilidades sociais de estudantes universitários em contextos interpessoais mais amplos ou de adolescentes em contextos também interpessoais mais abrangentes (DEL PRETTE; DEL PRETTE, 2009).

Em resumo, os itens do instrumento agora proposto medem exclusivamente os componentes acadêmico-sociais sociais no contexto universitário, o que o diferencia de outras medidas de habilidades sociais. Além disso, esta medida está mais voltada pra comportamentos e serve como parâmetro complementar para avaliar os indicadores relacionais da adaptação acadêmica a universidade.

Considerando que a quantidade de medidas de auto-relato que avaliam as habilidades e comportamentos sociais no contexto universitário é escassa, e que este instrumento constitui uma medida brasileira, além de seus fatores apresentarem boa consistência interna, recomenda-se a sua utilização em pesquisas acadêmicas que visem avaliar este construto em diferentes grupos e contextos educacionais de nível superior.

Estudos prospectivos poderiam verificar mais em profundidade a análise das características psicométricas do instrumento, utilizando processos de análises fatoriais confirmatórias e da análise de sua validade discriminante e convergente. Essas pesquisas certamente poderão contribuir para o aprimoramento do instrumento, bem como para o fortalecimento de sua capacidade preditiva.

Além disso, conforme salientam Gerk e Cunha (2006) seria interessante que escalas como estas fossem utilizadas pelas universidades em programas adaptação psicossocial dos estudantes que focasse a preparação para as mudanças que ocorrem após o ensino médio. O uso dessa escala em programas dessa natureza permitiria identificar tanto variáveis antecedentes como conseqüentes dos comportamentos acadêmico-sociais do estudante universitário, permitindo que ações fossem desenvolvidas não apenas para aumentar a adaptação psicossocial dos estudantes, como também para desenvolver tais comportamentos com prováveis impactos para o desempenho escolar e para o aproveitamento das diversas oportunidades de aprendizagem que o ambiente universitário oferece.

\section{Referências Bibliográficas}

ALMEIDA, L. S. Questionário de vivências acadêmicas para jovens universitários. Estudo de Construção e de Validação. Revista 
Galego-portuguesa de psicologia e educação, Braga, v. 2, n. 3, p. 113-130, 1998.

ALMEIDA, L. S.; FERREIRA, J. A. Questionário de vivências acadêmicas (QVA). Braga: Instituto de Educação e Psicologia, 1997.

ALMEIDA, L. S.; SOARES, A. P. C.; FERREIRA, J. A. G. Adaptação e rendimento acadêmico no ensino superior: fundamentação e validação de uma escala de vivências acadêmicas. Psicologia: teoria, investigação e prática, Braga, v. 1, p. 157-170, 1999.

ALMEIDA, L. S.; SOARES, A. P. Os estudantes universitários: sucesso escolar e desenvolvimento psicossocial. In: MERCURI, E. ; POLYDORE, S. A. J. (Eds.). Estudante universitário: características e experiências de formação. Taubaté: Cabral Editora e Livraria Universitária, 2004. (p. 15-40).

AZEVEDO, A.; FARIA, L. Impacto das condições pessoais e contextuais na transição do ensino secundário para o ensino superior.

Revista da UFP, Porto, v. 6, p. 257-269, 2001.

AZEVEDO, A. S.; FARIA, L. Motivação, sucesso e transição para o ensino superior. Psicologia, Lisboa, v. 20, n. 2, p. 69-93, 2001.

BANDEIRA, M.; COSTA, M, N.; DEL PRETTE, Z. A. P.; DEL PRETTE, A. ; GERK-CARNEIRO, E. Qualidades psicométricas do Inventário de Habilidades Sociais (IHS): estudo sobre a estabilidade temporal e a validade concomitante. Estudos de psicologia, Natal, v. 5, n. 2, p. 401-419, 2000.

BANDEIRA, M.; GUAGLIA, M. A. C. Habilidades sociais de estudantes universitários: identificação de situações sociais significativas. I nteração em psicologia, Curitiba, v. 9, n. 1, p. 45-55, 2005.

BANDEIRA, M. ; GUAGLIA, M. A. C. ; FREITAS, L. C. ; de SOUZA, A. M. ; COSTA, A. L. P.; GOMIDES, M. M. P.; LIMA, P. B. Habilidades interpessoais na atuação do psicólogo. Interação em psicologia, Curitiba, v. 10, n. 1, p. 139-149, 2006.

BANDEIRA, M.; DEL PRETTE, Z. A. P.; DEL PRETTE, A.; MAGALHÃES, T. Validação das escalas de habilidades sociais, comportamentos problemáticos e competência acadêmica (SSRS-BR) no ensino fundamental. Psicologia: teoria e pesquisa, Brasília, v. 5, n. 2, p. 271-28, 2009.

BARDIN, L. Análise de conteúdo. Lisboa: Edições 70, 1997.

BOLSONI-SILVA, A. T. Supervisão em habilidades sociais e seu papel na promoção deste repertório em estagiários de psicologia. Revista Brasileira de Terapias Cognitivas, Rio de Janeiro, v. 5, n. 1, p. 1832, 2009.

CABALLO, V. E. Los componentes condutales de la conduta assertiva. Revista de Psicologia General y Aplicada, Madrid, v. 37, n. 3, p. 473-486, 1982. 
CARELLI, M. J. G.; SANTOS, A. A. A. Condições temporais e pessoais de estudo em universitários. Psicologia Escolar e Educacional, Campinas, v. 2, n. 3, p. 265-278, 1998.

CASTILLA, M. E. Evaluation of Social Abilities in Educational Contexts: A Challenge for Students with Communication Difficulties. In Book series: Advances in Learning and Behavioral Disabilities, Volume 20. Bingley-WA: Emerald Group Publishing Limited, 2007. (p. 241-263).

CUNHA, S. A inteligência e as habilidades sociais na adaptação de alunos ao curso superior: um estudo com alunos do $1^{\circ}$ ano do Instituto Militar de Engenharia. 2004. Dissertação (Mestrado) Universidade Gama Filho: Rio de Janeiro.

CUNHA, S. M.; CARRILLO, D. M. O processo de adaptação ao ensino superior e o rendimento acadêmico, Psicologia Escolar e Educacional, Campinas, v. 9, n. 2, p. 215-224, 2005.

DEL PRETTE, A.; DEL PRETTE, Z. A. P. Análise de repertório assertivo em estudantes de psicologia. Revista de Psicologia, Fortaleza, v. 1, n. 1, p. 15-24, 1983.

DEL PRETTE, A.; DEL PRETTE, Z. A. P.; BARRETO, M. C. M. Análise de um inventário de habilidades sociais. Psicologia: Teoria e Pesquisa, Brasília, v. 3, n. 14, p. 219-288, 1988.

- Habilidades sociales en la formación del psicólogo: Análisis de un programa de intervención. Psicología Conductual, Granada, v. 7, p. 27-47, 1999.

DEL PRETTE, Z. A. P.; DEL PRETTE, A. Inventário de Habilidades Sociais (IHS -Del Prette): manual de aplicação, apuração e interpretação, 2a. ed. São Paulo: Casa do Psicólogo. 2001.

- Avaliação de habilidades sociais de crianças com um inventário multimídia: indicadores sociométricos associados a freqüência versus dificuldade. Psicologia em Estudo, Maringá, v. 7, n. 1, p. 39-49, 2002.

DEL PRETTE, A.; DEL PRETTE, Z. A. P. No contexto da travessia para o ambiente de trabalho: treinamento de habilidades sociais com universitários. Estudos de Psicologia, Natal, v. 8, n. 3, p. 413-420, 2003.

DEL PRETTE, Z. A. P. Psicologia escolar e educacional: saúde e qualidade de vida. Campinas: Alínea. 2003.

DEL PRETTE, Z. A. P.; DEL PRETTE, A.; BARRETO, M. C. M.; BANDEIRA, M.; RIOS-SALDANÃ, M. R.; ULIAN, A. L. A. O.; GERKCARNEIRO, E.; FALCONE, E. M. O.; VILLA, M. B. Habilidades sociais de estudantes de Psicologia: um estudo multicêntrico. Psicologia: Reflexão e Crítica, Porto Alegre, v. 17, n. 3, p. 341-350, 2004.

DEL PRETTE, Z. A. P. Psicologia das habilidades sociais na infância: teoria e prática. Petrópolis, RJ: Vozes. 2005.

DEL PRETTE, Z. A. P.; VILLA, M. B.; FREITAS, M. G.; DEL PRETTE, A. Estabilidade temporal do Inventário de Habilidades Sociais Conjugais 
(IHSC). Avaliação Psicológica, Porto Alegre, v.7, n. 1, p. 67-74, 2008.

DEL PRETTE, A.; DEL PRETTE, Z. A. P. I nventário de Habilidades Sociais para Adolescentes (IHSA-Del-Prette): Manual de aplicação, apuração e interpretação. São Paulo: Casa do Psicólogo, 2009.

ENZMANN, D. RanEigen: A program to determine the parallel analysis criterion for the number of principal components. Applied Psychological Measurement, Califórnia, v. 21, p. 232, 1977.

FALCONE, E. M. O.; FERREIRA, M. C.; LUZ, R. C. M.; FERNANDES, C. S.; FARIA, C. A. ; D'AUGUSTIN, J. F.; SARDINHA, A.; PINHO, V. D. Inventário de Empatia (I. E.): Desenvolvimento e validação de uma medida brasileira. Avaliação Psicológica, Porto Alegre, v. 7, n. 3, p. 321-334, 2008.

FERREIRA, J. A.; ALMEIDA, L. S.; SOARES, A. P. C. Adaptação acadêmica em estudante do $1^{0}$ ano: diferenças de gênero, situação do estudante e curso. Psico-USF, Itatiba, v. 6, n. 1, p. 1-10, 2001.

FURTADO, E. S.; FALCONE, E. M. O.; CLARK, C. Avaliação do estresse e das habilidades sociais na experiência acadêmica de estudantes de medicina de uma universidade do Rio de Janeiro. Interação em Psicologia, Curitiba, v. 7, n. 2, p. 43-51, 2003.

GERK, E.; CUNHA, S. M. As habilidades sociais na adaptação de estudantes ao ensino superior. In: Bandeira, M., Del Prette, Z. A. P., Del Prette, A. (Orgs.). Estudos sobre habilidades sociais e relacionamento interpessoal São Paulo: Casa do Psicólogo, 2006. (p. 181-198).

GRANADO, J. I. F.; SANTOS, A. A. A.; ALMEIDA, L. S.; SOARES, A. P.; GUISANDE, M. A. Integração académica de estudantes universitários: Contributos para a adaptação e validação do QVA-r no Brasil. Psicologia e Educação, Covilhã, v. 12, n. 2, p. 31-43, 2005. IGUE, E. A.; BARIANI, I. C. D.; MILANESI, P. V. B. Vivência acadêmica e expectativas de universitários ingressantes e concluintes. PsicoUSF, Itatiba, v. 13, n. 2, p. 155-164, 2008.

NASCIMENTO, A. R. A.; MENANDRO, P. R. M. Análise lexical e análise de conteúdo: uma proposta de utilização conjugada. Estudos e Pesquisas em Psicologia, Rio de Janeiro, v. 6, n. 2, p. 72-88, 2006.

PACHANE, G. G. A experiência universitária e sua contribuição ao desenvolvimento pessoal do aluno. In: MERCURI, E.; POLYDORE, S. A. J. (Orgs.). Estudante universitário: características e experiências de formação Taubaté: Cabral, 2003. (p. 155-186).

PASCARELLA, E. T.; TERENZINI, P. Twenty years of research on college students: Lessons for future reseach, Volume 1. San Francisco: Jossey-Bass, 1991.

PASCARELLA, E. T.; TERENZINI, P. T. How College Affects Students: a third decade of research. Volume 2. San Francisco: 
Jossey-Bass, 2005.

PARKER, J. D. A.; SUMMERFELDT, L. J.; HOGAN, M. J.; MAJESKI, S. A. Emotional intelligence and academic success examining the transition from high school to university. Personality and I ndividual Differences, Londres, v. 36, p. 163-172, 2004.

PERRENOUD, Ph. Construir as competências desde a escola. Porto Alegre: Artmed Editora.1999.

PERRENOUD, Ph. Dez novas competências para ensinar. Porto Alegre: Artmed Editora. 2000.

PERRENOUD, Ph. Porquê construir competências a partir da escola? Desenvolvimento da autonomia e luta contra as desigualdades. Porto: ASA Editores, 2001

PERRENOUD, Ph.; GATHER THURLER, M.; DE MACEDO, L.; MACHADO, N. J.; ALLESSANDRINI, C. D. As competências para ensinar no século XXI. A Formação dos Professores e o Desafio da Avaliação. Porto Alegre: Artmed Editora, 2002.

PIRES, H. S.; ALMEIDA, L.; FERREIRA, J. A. Questionário Acadêmico de Vivências Acadêmicas (QVA) aos estudantes universitários das PALOP. In: SOARES, A. P.; OSÓRIO, A.; CAPELA, J. V.; ALMEIDA, L. S.; VASCONCELOS, R. M.; CAÍRES, S. M. (Eds.). Transição para o ensino superior. Braga: Universidade de Minho, 2000. (p. 119127).

POLYDORO, S. A. J.; PRIMI, R.; SERPA, M. de N. da F.; ZARONI, M. M. H.; POMBAL, K. C. P. Desenvolvimento de uma escala de integração ao ensino superior. Psico-USF, Itatiba, v. 6, n. 1, p. 1117, 2001.

POLYDORO, S. A. J. O trancamento de matrícula na trajetória acadêmica do universitário: condições de saída e de retorno à universidade. 2000. Tese (Doutorado) - Faculdade de Educação, Universidade Estadual de Campinas, Campinas, São Paulo.

SÁ, A. P. Propriedades psicométricas de uma escala de auto-eficácia acadêmica e suas relações com desempenho estudantil e interação social. Revista Brasileira de Terapias Cognitivas,Rio de Janeiro, v. 2, n. 2, p. 61-72, 2006.

SADALLA, A. M. F. A. Com a palavra a professora: Suas crenças, suas ações. Campinas: Alínea, 1998.

SANTOS, S. M. As responsabilidades da universidade no acesso ao ensino superior. In: SOARES, A. P.; OSÓRIO, A; CAPELA, J. V.; ALMEIDA, L. S.; VASCONCELOS, R. M.; CAÍRES, S. M. (Eds.). Transição para o ensino superior. Braga: Universidade de Minho, 2000. (p. 69-78).

SANTOS, A. A. A.; SUEHIRO, A. C. B.; OLIVEIRA, E. Z.; CUNHA, S. M.; CARROLLO, D. M. Avaliação da integração acadêmica no ensino superior: Estudo com estudantes de engenharia. Psicologia e Educação, Covilhã, v. 5, n. 2, p. 79-89, 2006.

SOARES, A. P.; ALMEIDA, L. S.; DINIZ, A.; GUISANDE, M. A. Modelo 
multidimensional de ajustamento de jovens ao contexto Universitário (MMAU): Validacão com alunos de ciências e tecnologias versus alunos de ciências sociais e humanas. Análise Psicológica, Lisboa, v. 1 , p. 15-28, 2006.

SOARES, A. P.; GUISANDE, A. M.; ALMEIDA, L. S.; PÁRAMO, F. M. Academic achievement in first-year Portuguese college students: The role of academic preparation and learning strategies. I nternational J ournal of Psychology, Montreal, v. 44, n. 3, p. $204-212,2008$. SOARES, A. B.; NAIFF, L. A. M.; FONSECA, L. B.; CARDOSO, A.; BALDEZ, M. O. Estudio comparativo de habilidades sociales y variables sócio demográficas de profesores. Psicologia: Teoria e Prática, São Paulo, v. 11, n. 1, p. 35-49, 2009.

SOARES, A. B.; POUBEL, L. N.; MELLO, T. V. S. Habilidades sociais e adaptação acadêmica: um estudo comparativo em instituições de ensino público e privado, Aletheia, Canoas, v. 29, p. 27-42, 2009.

VENDRAMINI, C. M. M.; SANTOS, A. A. A.; POLYDORO, S. A. J.; SBARDELINI, E. T. B.; SERPA, M. N. F.; NATÁRIO, E. G. Construção e validação de uma escala sobre avaliação da vida acadêmica (EAVA). Estudos de Psicologia, Natal, v. 9, n. 2, p. 259-268, 2004.

VILLAR, J. D. Adaptação de questionário de vivencia universitária com estudantes de Arquitetura e Engenharia. 2003. Dissertação de mestrado, Itatiba: Universidade São Francisco.

VILLASBOAS, A. C. V. B.; SILVEIRA, F. F.; BOLSONI-SILVA, A. T. Descrição de efeitos de um procedimento de intervenção em grupo com universitários: um estudo piloto. Interação em Psicologia, Curitiba, v. 9, p. 321 - 330, 2005.

ZHANG, L. F. University students' learning approaches in three cultures: An investigation of Bigg's 3P model. The Journal of Psychology, Greenville, NC, v. 134, p. 37-55, 2000.

ZHENG, J. L.; KEVIN, L.; SAUNDERS; P.; SHELLEY, M. C. Predictors of academic success for freshmen residence hall students. J ournal of College Student Development, Nashville, TN, v. 43, p. 267-273, 2002.

\section{Endereço para correspondência}

Adriana Benevides Soares

Rua Marechal Deodoro, 263, Centro, CEP 24030-060, Rio de Janeiro, RJ, Brasil

Endereço eletrônico: adribenevides@gmail.com

Luciana Mourão

Rua Marechal Deodoro, 263, Centro, CEP 24030-060, Rio de Janeiro, RJ, Brasil

Endereço eletrônico: mourao.luciana@gmail.com

Thatiana Valory dos Santos Mello

Praça Floriano, 51, Centro, CEP 2402-340. Rio de Janeiro, RJ, Brasil

Endereço eletrônico: thatianavalory@gmail.com

Recebido em: 20/07/2010

Reformulado em: 13/09/2010

Aceito para publicação em: 16/09/2010 
Adriana Benevides Soares, Luciana Mourão, Thatiana Valory dos Santos Mello Estudo para a construção de um instrumento

Acompanhamento do processo editorial: Deise Mancebo

\section{Notas}

*Psicóloga. Doutora em Psicologia Cognitiva pela Universidade de Paris XI; Docente da graduação e da pós-graduação da Universidade do Estado do Rio de Janeiro UERJ; Docente da Universidade Salgado de Oliveira - UNIVERSO, Rio de Janeiro.

**Comunicadora social e administradora; Docente de graduação da Associação Internacional de Educação Continuada - AIEC; Docente da Universidade Salgado de Oliveira - UNIVERSO.

***Psicóloga; Mestre em Psicologia pela Universidade Salgado de Oliveira; Docente da Universidade Estácio de Sá - UNESA. 